\title{
Current Concepts: Laser Treatment of Adult Vascular Lesions
}

\author{
Tomi L. Wall, M.D. ${ }^{1}$
}

\section{ABSTRACT}

Patients frequently seek treatment of cutaneous vascular lesions for both medical and cosmetic reasons. Advances in the use of lasers and light sources enable physicians to effectively treat vascular lesions that were previously untreatable. Given the plethora of devices available, selecting the appropriate laser system and treatment parameters can be confusing for the laser surgeon. This article reviews current concepts in the treatment of adult vascular conditions. It highlights the different types of lasers and the most common vascular lesions amenable to therapy. Also, the article offers several pearls regarding selection of the laser and of the treatment parameters.

KEYWORDS: Laser, vascular lesions, treatment, review, skin, telangiectasia, port wine stain, IPL

Vascular lesions are often a cause of great concern to patients. Treatment is sought for a variety of reasons including cosmesis, pain, bleeding, or disfigurement. In the late 1960 s and early 1970 s, physicians began treatment of vascular anomalies with continuous-wave lasers such as the argon laser. Although these lasers were effective, they often caused unacceptable side effects such as scarring and permanent dyspigmentation. These outcomes limited the widespread use of this modality. In the 1980s, Anderson and Parrish described the theory of selective photothermolysis and revolutionized the treatment of vascular lesions. ${ }^{1}$ Determining the appropriate wavelength, pulse duration, and fluence enables physicians to selectively target hemoglobin within blood vessels without damaging the surrounding tissue. As a result, side effects such as dyspigmentation and scarring are minimal. This article reviews the types of lasers currently available to treat common adult vascular lesions, describes treatment strategies, and highlights methods to minimize adverse effects.

\section{BASIC CONCEPTS}

The ultimate goal of laser treatment is to induce vessel wall damage through destruction of hemoglobin while minimizing injury to adjacent structures. Vessel destruction occurs either through photomechanical or photothermal means. When short pulse-widths (or pulse durations) are used, photomechanical damage occurs. This causes intravascular cavitation, vessel wall rupture, and hemorrhage. Clinically, one sees purpura

\footnotetext{
${ }^{1}$ Department of Dermatology, Wellman Laboratories of Photomedicine, Harvard Medical School, Massachusetts General Hospital, Boston, Massachusetts.

Address for correspondence and reprint requests: Tomi L. Wall, M.D., 3300 Webster Street, Suite 1106, Oakland, CA 94609.
}

Lasers in Plastic Surgery; Guest Editor, Ramsey F. Markus, M.D. Semin Plast Surg 2007;21:147-158. Copyright (C) 2007 by Thieme Medical Publishers, Inc., 333 Seventh Avenue, New York, NY 10001, USA. Tel: +1(212) 584-4662.

DOI 10.1055/s-2007-991183. ISSN 1535-2188. 
after treatment. With longer pulse-widths or stuttered pulses, photothermal damage occurs. This causes slow heating of the vessel, intravascular coagulation, and collagen contraction. Clinically, one sees immediate blanching or subtle darkening of the vessel followed by subsequent erythema and edema.

The first consideration in the selection of a laser is to determine the spectral absorption peaks of the target chromophore. In the case of vascular lesions, this is deoxyhemoglobin or oxyhemoglobin. Hemoglobin shows absorption peaks in the blue, green, and yellow bands $(418,542$, and $577 \mathrm{~nm})$ as well as a peak further out in the near-infrared portion of the spectrum $(700$ to $1100 \mathrm{~nm})$. Laser systems used to treat vascular lesions emit wavelengths near these peaks. Melanin is a strong competing chromophore at shorter wavelengths. Therefore, it is preferable to select longer wavelengths whenever possible. This is especially important for patients with darker skin, such as those with Fitzpatrick skin types IV to VI. If shorter-wavelength lasers are used, melanin may absorb the laser energy intended for hemoglobin and result in dyspigmentation. The wavelength also determines depth of penetration through the epidermis and dermis. Longer wavelengths may be preferred for deeper vascular lesions such as leg veins, whereas shorter wavelengths may be suited for superficial vascular lesions such as facial telangiectasias.

Once the appropriate laser is selected, it is important to choose the correct parameters. These include the pulse duration, fluence, and spot size. The pulse duration is determined by the thermal relaxation time (TRT) of the target. Essentially, this is defined as the time required for the heated tissue to lose about half of its heat. It is roughly proportional to the square of the target's diameter. Therefore, the TRT of larger vessels such as leg veins will range in hundreds of milliseconds, whereas the TRT of smaller vessels such as telangiectasias will range in tens of microseconds. A laser pulse that is shorter than or equal to the TRT will cause damage limited to the vessel. A laser pulse that is greater than the TRT will cause heat diffusion outside of the vessel into surrounding structures, causing scarring or textural changes. Fluence is defined as the energy per unit area. It is inversely proportional to the fraction of light absorbed by the target. A higher fluence will be necessary if a target is deep within the dermis or the wavelength that the laser emits is weakly absorbed. Spot size should approximate the diameter of the target vessel to minimize surrounding damage. Larger spot sizes tend to have less scattering of the laser beam and deeper dermal penetration.

In treatment of all vascular lesions, cooling of the skin surface is crucial to minimize epidermal damage and allow maximal fluences. These cooling methods can be found within the laser system or can be externally applied. Integrated cooling devices include chilled tips, cooled glass chambers, or pulsed cryogen sprays. ${ }^{2}$ External cooling devices include forced-air cooling, ice packs, or cool gels applied to the surface of the skin prior to treatment.

\section{TYPES OF VASCULAR LASERS}

\section{Flashlamp-Pumped Pulsed Dye Laser}

Pulsed dye lasers (PDLs) use a rhodamine dye that is dissolved in a solvent and pumped by a flashlamp. This laser energy is delivered to tissue by fiberoptics. Initial PDLs emitted a wavelength of $577 \mathrm{~nm}$ and had a fixed pulse duration of 0.45 milliseconds. Because of their limited depth of penetration, later models were developed with longer wavelengths and variable pulse durations. Current PDLs emit wavelengths ranging from 585 to $600 \mathrm{~nm}$ with pulse widths ranging from 0.45 to 40 milliseconds. Spot sizes can vary from 3 to $12 \mathrm{~mm}$ in diameter. Maximal energy fluences of $40 \mathrm{~J} / \mathrm{cm}^{2}$ are available, depending on the spot size. It is perhaps the most specific laser currently available for the treatment of superficial vascular lesions. Advantages of the PDL are that it can effectively treat many types of vascular lesions such as telangiectasias, hemangiomas, and rosacea. It is the treatment of choice for port wine stains. With the variable pulse durations, the operator can choose the clinical end point to be purpura or erythema depending on the lesion to be treated and patient preference.

\section{Potassium Titanyl Phosphate Laser}

Potassium titanyl phosphate (KTP) lasers are quasicontinuous lasers that use a neodymium:yttriumaluminum-garnet crystal frequency doubled with a KTP crystal to emit a wavelength of $532 \mathrm{~nm}$. Pulse durations range from 1 to 150 milliseconds, fluences up to $240 \mathrm{~J} / \mathrm{cm}^{2}$, and spot sizes up to $5 \mathrm{~mm}$. It produces energy pulses with small spot sizes and as a result can be used in the treatment of smaller vessels such as facial telangiectasias. Scanning devices can be attached to some of these systems to deliver non-overlapping pulses over larger areas. The advantage of the KTP is that target vessels are not ruptured, leading to much less purpura. In addition, it is smaller and more easily portable than other lasers. The disadvantage is that only patients with Fitzpatrick skin types I to III are candidates because there is significant melanin absorption at this wavelength. Patients with darker skin types will often have long-lasting dyspigmentation, blistering, or significant crusting. 


\section{Long-Pulse Neodymium:Yttrium-Aluminum- Garnet Laser}

In neodymium:yttrium-aluminum-garnet (Nd:YAG) lasers, the active medium is a YAG crystal doped (the crystal is grown in the presence of an impurity) with 1 to $3 \%$ neodymium ions. Nd:YAG lasers typically produce light in the near-infrared range of the spectrum at a wavelength of $1064 \mathrm{~nm}$. Pulse durations for the long-pulsed Nd:YAG may range up to 300 milliseconds and the fluences up to $900 \mathrm{~J} / \mathrm{cm}^{2}$. Spot sizes range from 3 to $10 \mathrm{~mm}$. It is primarily used for laser hair removal; however, Nd:YAG lasers, with their longer wavelengths and pulse durations, may be useful for deep and resistant vessels. The Nd:YAG laser can be used on patients with Fitzpatrick skin types IV to VI because there is much less melanin as a competing chromophore at this wavelength; however, care must be taken when using this laser due to higher incidences of scarring and blistering when compared with the PDL. When Nd:YAG lasers are manufactured to emit $532 \mathrm{~nm}$, this is done with a KTP crystal that frequency doubles and halves the wavelength to $532 \mathrm{~nm}$. Uses for the $532-\mathrm{nm} \mathrm{Nd:YAG}$ are similar to uses mentioned in the section above on KTP.

\section{Long-Pulse Alexandrite Laser}

Long-pulsed millisecond alexandrite lasers emit a wavelength of $755 \mathrm{~nm}$ and have pulse durations varying from 3 to 40 milliseconds. Spot sizes are available from 6 to $18 \mathrm{~mm}$, and energy output is maximal at $100 \mathrm{~J} / \mathrm{cm}^{2}$. It is primarily used for laser hair removal and treatment of pigmented lesions. Although the main chromophore absorbed at this wavelength is melanin, hemoglobin also has a small but significant peak of absorption. The alexandrite penetrates to depths of 2 to $5 \mathrm{~mm}$. These characteristics make this laser useful for various deeper and more resistant vascular lesions.

\section{Diode Laser}

The diode laser is perhaps the most efficient light source. It is a small, solid-state semiconductor with no moving parts and minimal energy requirements. The laser light is delivered by fiberoptics. It is inexpensive, compact, and portable. Wavelengths can vary and include 532, 800, $810-, 940$, and $980 \mathrm{~nm}$. The quasi-continuous wave 532-nm diode can produce laser pulses of 15 to 150 milliseconds with energy outputs up to $110 \mathrm{~J} / \mathrm{cm}^{2}$. The smaller spot sizes of 200 to $1400 \mu \mathrm{m}$ allow for treatment of smaller telangiectasias. The 532-nm diode is advantageous for Fitzpatrick skin types I to III for reasons similar to the KTP laser. The longer-wavelength diode lasers are used for laser hair removal but can be useful for deeper vascular lesions.
Broadband Light Sources: Intense Pulse Light

Incoherent polychromatic pulsed light sources such as intense pulse light (IPL) utilize a xenon flashlamp powered by a capacitor bank. The flashlamps are surrounded by water, which cools and cuts down on the longer infrared emissions. IPLs emit wavelengths between 515 and $1000 \mathrm{~nm}$; however, specific cutoff filters with various wavelengths block out shorter wavelengths. Initial IPL systems were bulky, inefficient, unpredictable, and greatly operator dependent. In some cases, holding the hand piece several millimeters away from the skin varied the fluence significantly; however, newer systems are now much more consistent and accurate. The advantage of the IPL is its versatility, which allows treatment of both vascular and pigmented lesions. In addition, the larger spot size allows for efficient treatment of extensive lesions. The disadvantages are slow repetition rates $(0.3$ to $1 \mathrm{~Hz})$ and the lack of maneuverability.

\section{Combination Lasers}

Recently, combination lasers and light sources have emerged that combine two different lasers or light/energy sources. Examples of systems that combine two lasers include the1064-nm and 595-nm (Synergy; Cynosure, Chelmsford, MA), the 532-nm and 1064-nm (Gemini; Laserscope, San Jose, CA), and the 532-nm and 940-nm (VariLite; Iridex, Mountain View, CA). A system that combines Nd:YAG (clear scan) and broadbard light IPL also exists (Sciton, Palo Alto, CA). These may have a role in offices where space is a factor, and they may allow for treatment of various types of vascular lesions in one setting. There are no head-to-head studies comparing the efficacy of these combined systems with traditional lasers or energy sources.

\section{TYPES OF ADULT VASCULAR LESIONS AMENABLE TO LASER THERAPY}

\section{Telangiectasias (Including Rosacea,} Poikiloderma of Civatte, and Spider Angioma)

Telangiectasias are commonly occurring cutaneous vessels that typically measure 0.1 to $1.0 \mathrm{~mm}$ in diameter. Significant sun exposure, hormonal factors, or genetic predisposition may all play a role in telangiectasia development. Associated acquired or genetic causes of telangiectasias include primary cutaneous disorders such as rosacea or systemic diseases such as collagen vascular disorders (e.g, CREST [calcinosis cutis, Raynaud phenomenon, esophageal motility disorder, sclerodactyly, and telangiectasia syndrome]). Telangiectasias may also be a component of cutaneous conditions such as poikiloderma of Civatte. Histologically, vessels represent dilated postcapillary venules, capillaries, or arterioles 


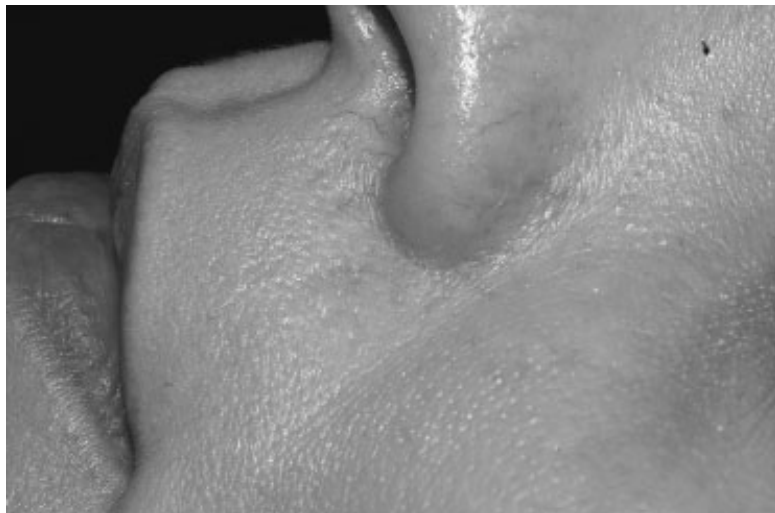

Figure 1 Nasolabial fold telangiectasias before treatment.

with thickening of the vessel wall. Depths range from 200 to $300 \mu \mathrm{m}$. Regardless of the underlying cause, all forms of telangiectasias can be treated successfully with laser surgery. Studies show improvement with many different lasers, including the $577-\mathrm{nm} \mathrm{PDL}^{3}, 585-$ to 600-nm PDL, ${ }^{4}$ 532-nm diode, $940-\mathrm{nm}$ diode, $532-\mathrm{nm}$ $\mathrm{KTP},{ }^{5} 532-\mathrm{nm} \mathrm{Nd:YAG},{ }^{6}$ and light sources such as the IPL. ${ }^{7}$ Long-pulsed 1064-nm Nd:YAG lasers have also been used successfully for larger periorbital and temporal veins, but much higher fluences are necessary at this wavelength. ${ }^{8}$

If the telangiectasia is small in diameter, lasers such as the 532-nm KTP with small spot sizes are useful to trace the individual vessels. Large-diameter vessels require lasers with longer wavelengths or variable pulsewidths such as the PDL or long-pulsed 1064-nm $\mathrm{Nd}$ :YAG for adequate destruction. Telangiectasias in specific locations such as the nasolabial fold tend to be more resistant to treatment, so lasers with larger pulse durations and higher fluences such as the 940-nm diode or PDL are most useful. Treatment on the neck or chest should be performed with reduced fluences to minimize scarring. If lesions are diffuse or extensive, PDL or IPL with larger spot sizes or KTP with scanning devices may be more efficient. For patients who desire no "downtime" (or purpura), newer stuttered PDLs with variable pulse-widths, diode, KTP, or IPL can be used.

In general, focal lesions may resolve with one or two treatments with any of these devices, whereas extensive lesions may require multiple treatments. Subpurpuric doses tend to require more frequent treatments. Treatments are typically spaced 4 to 6 weeks apart. Starting fluences vary from laser to laser. It is prudent to start with lower fluences recommended by the manufacturer, perform test spots, and examine the skin for the immediate desired clinical end point. With the PDL, the desired clinical end point is immediate vessel disappearance followed by light pur-

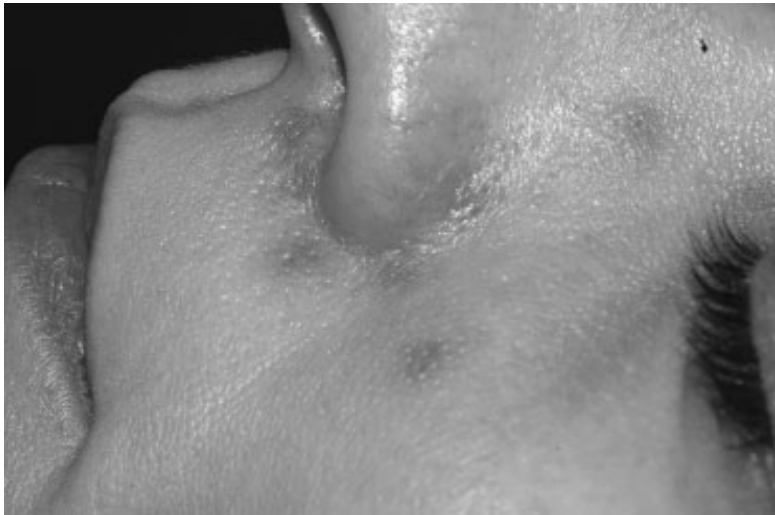

Figure 2 Nasolabial fold telangiectasias immediately after treatment with PDL. Note immediate purpura visible especially in the infranasal region.

pura within minutes (Figs. 1 and 2). A steel-gray color indicates that the fluence is too high, and too much heat diffusion may have taken place. In the case of KTP, IPL, or with PDLs set to subpurpuric settings, the desired clinical end point is immediate vessel blanching or subtle graying with subsequent erythema (Figs. 3 and 4).

In the treatment of rosacea, the 585-nm PDL with a $5-$ or $7-\mathrm{mm}$ spot size, fluences between 5 and $7 \mathrm{~J} / \mathrm{cm}^{2}$, and pulse duration of 0.45 milliseconds has been shown to produce good to excellent reduction in telangiectasia, erythema, and overall appearance as well as a decrease in papules and pustules. ${ }^{9}$ In addition, a decrease in oral antibiotic dose was possible after 1 to 3 PDL treatments in some cases. ${ }^{10} \mathrm{Im}^{-}$ provement of stinging and burning as well as quality of life has been noted. ${ }^{11}$ These original studies resulted in purpura in many cases, which resolved within 5 to 14 days. Because of the desire of patients for treatments without purpura, subpurpuric doses with the 595-nm PDL using longer pulse durations (10 to 40 milliseconds) were compared with purpuric settings. These studies show that even with multiple passes, purpuric doses resulted in better clearing of vessels than subpurpuric doses. ${ }^{12-14}$ KTP or diode has been successful in the treatment of clinically visible telangiectasias, but these shorter-wavelength lasers show little to no improvement of background erythema. IPL has been used with some success, but results are modest in comparison with the PDL. ${ }^{15,16}$ The IPL, because of its polychromatic nature, may be advantageous in patients with diffuse background erythema.

Treatment of poikiloderma of Civatte, or any treatment on the neck and chest, should be performed with caution using lower fluences and larger spot sizes to minimize the potential adverse effects such as textural changes and pigmentary alterations. Keep in mind that it is important to treat the pigmentary alterations 


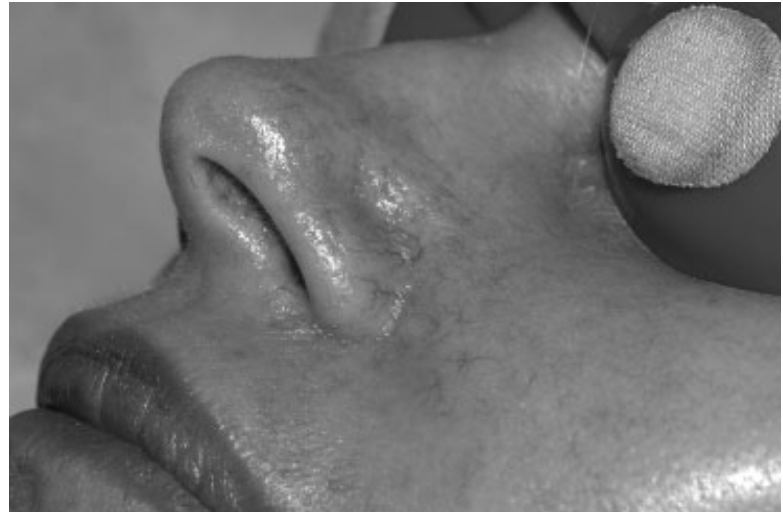

Figure 3 Nasolabial fold telangiectasias before treatment.

seen with this condition as well as the telangiectatic component for successful clearance. Use of the PDL with lower fluences of 6 to $7 \mathrm{~J} / \mathrm{cm}^{2}$ and spot sizes of $5 \mathrm{~mm}$ has been shown to be effective ${ }^{17}$; however, scarring was noted in some cases with fluences greater than $6 \mathrm{~J} / \mathrm{cm}^{2} .{ }^{18}$ Mottling of the skin can be seen due to the circular spot size if appropriate overlap is not used. Studies have shown that IPL may be effective because of its larger spot size and the fact that it can simultaneously target the vascular and pigmented components. ${ }^{19,20}$

Spider angiomas are a cluster of dilated capillaries radiating from a central feeding arteriole. When treating with lasers, it is important to treat the central arteriole along with all of the superficial branches to avoid recurrence. Treatment of spider angiomas has been successful with the PDL, ${ }^{21,22}$ although the 532-nm Nd:YAG and IPL have been used as well. The IPL may be unwieldy because of its larger spot size. Keep in mind that purpuric doses are often needed for complete resolution, and shorter pulsewidths are often more effective. Use of diascopy of arrest flow in the central arteriole in spider telangiectasias or to treat the deeper component of papular vessels may be helpful.

\section{Capillary Malformation/Port Wine Stain}

Port wine stain (PWS) occurs in 0.3 to $0.5 \%$ of newborns initially as a light-pink macule or patch anywhere on the body. ${ }^{23}$ Histologically, there are an increased number of ectatic vessels in the superficial papillary or upper reticular dermis. S100 staining shows a paucity of nerves, which may account for altered modulation of vascular tone and gradual ectasia. ${ }^{24}$ Over time, the color may deepen and become dark-red or violaceous with associated hypertrophy and nodularity in two thirds of patients by age $46 .^{25}$ Treatments are preferred in childhood to minimize

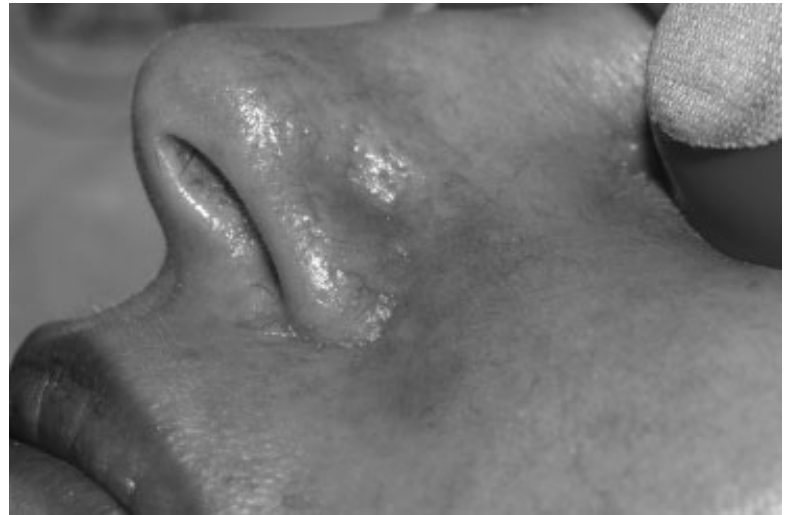

Figure 4 Nasolabial fold telangiectasias immediately after treatment with IPL. Note erythema and slight edema of vessels in the alar groove.

psychological trauma and to prevent hypertrophy; however, a favorable response to treatment-both physically and psychologically-is not dependent on age. $^{26}$ Studies of adults with PWS show that those who initially had difficulty forming interpersonal relationships with members of the opposite sex had marked improvement in psychological health after successful treatment. ${ }^{27,28}$

When evaluating a patient with PWS, there are several important factors to consider. Location and size of the PWS is important, given that head (in particular, the central forehead and peripheral face) and neck lesions respond better than lesions at other sites such as the lower extremities or trigeminal V2 distribution. ${ }^{29,30}$ Any areas of concern such as scaling or bleeding within the PWS of an adult with a history of previous radiation therapy in that region warrants a biopsy to rule out underlying basal cell carcinoma. ${ }^{31-34}$ Areas of scarring from prior laser treatments, hypertrophy, nodularity, or associated pyogenic granulomas should be noted, and appropriate evaluation for underlying systemic involvement must be undertaken in cases of suspected syndromes such as Sturge-Weber or Klippel-Trenaunay-Weber.

Treatment of choice for most patients currently is with the pulsed dye laser. Various studies cite that $\sim 65 \%$ of patients treated with PDL will achieve 50 to $90 \%$ lightening of their PWS, and $15 \%$ of patients will achieve greater than $90 \%$ lightening. ${ }^{35,36}$ Multiple treatment sessions are necessary for improvement of the lesions, and series of anywhere from 4 to 12 treatments spaced 4 to 6 weeks apart are generally recommended. Patients with a PWS present in the nonfacial regions should be informed that a higher number of treatments and more adverse effects may be possible. PDL with wavelengths of 585 to $600 \mathrm{~nm}$ and spot sizes of 7 to $10 \mathrm{~mm}$ with fluences of 8 to $12 \mathrm{~J} / \mathrm{cm}^{2}$ are used. Pulse durations vary from 0.45 to 10 millisecond depending on the diameter of the vessels. The 


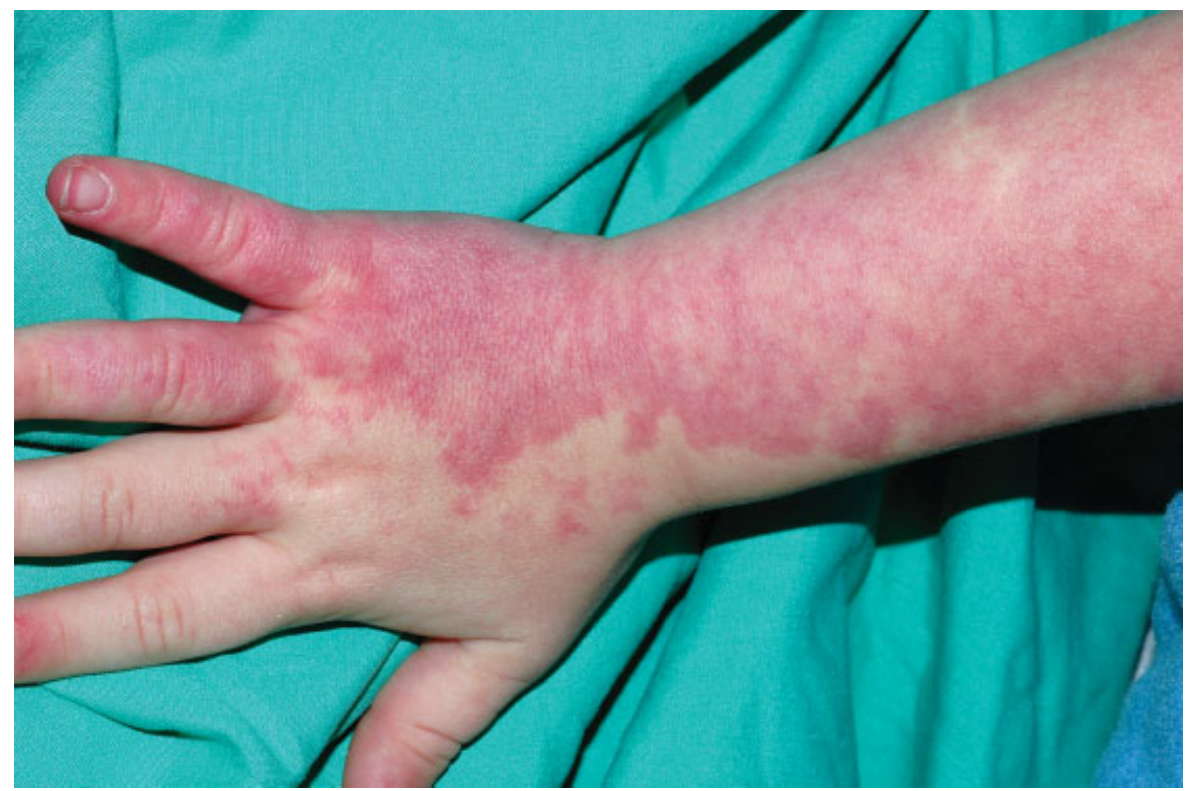

Figure 5 PWS before treatment.

desired clinical end point with all PWS is light purpura seen immediately or up to several minutes after treatment (Figs. 5 and 6). If development of a steel-gray purpura is seen, the fluence may be too high, and higher risk of scarring or blistering may result (Fig. 7).

Reported adverse effects are typically low with the development of pulsed lasers and epidermal cooling devices. The most common side effect is purpura, which can last up to 14 days in some cases. Non- purpuric treatment often results in less efficacious treatment. Transient hyperpigmentation can be seen, which usually clears after several months. Risk of atrophic and hypertrophic scarring is less than $1 \%$ with the PDL. ${ }^{37}$

Mature, recurrent, or resistant PWS lesions may pose special challenges to the laser surgeon. Longer wavelengths, longer pulse-widths, and higher fluences can all be utilized to attain deeper penetration and target larger vessel size. ${ }^{38,39}$ IPL has been

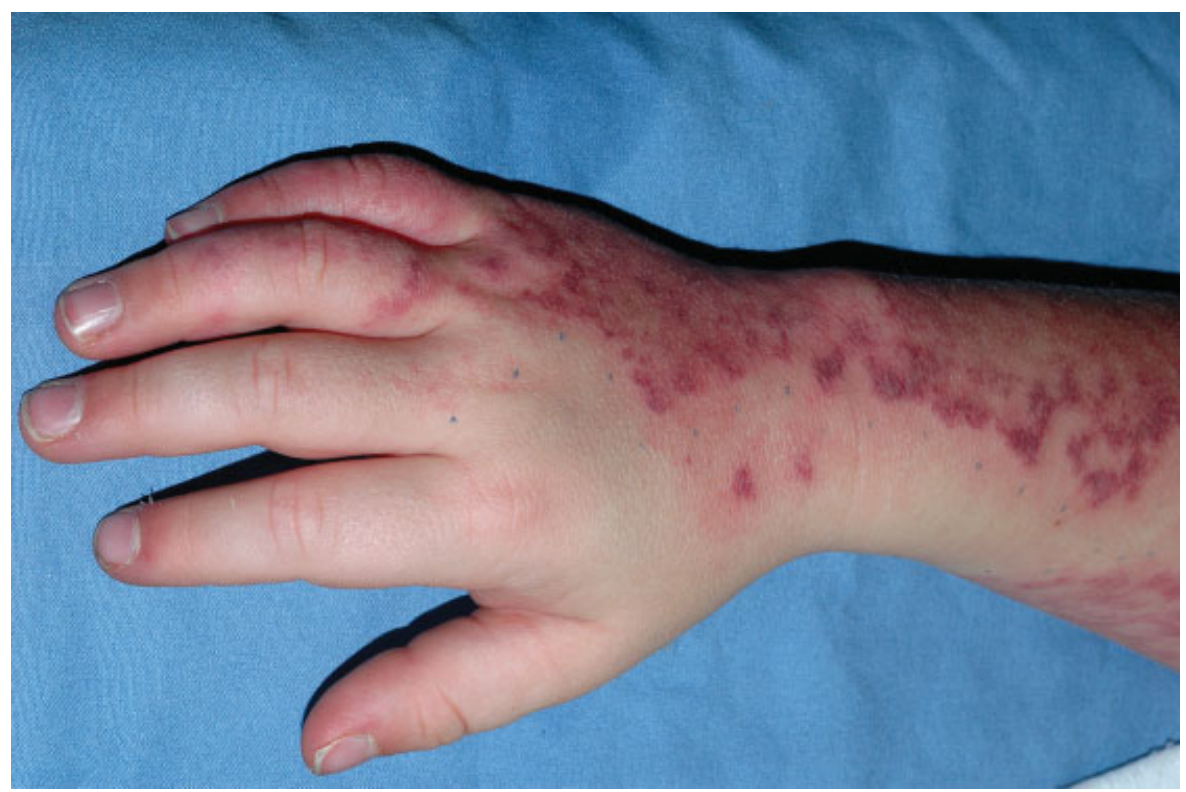

Figure 6 PWS several minutes after treatment with PDL. Note the desired light purpura. 


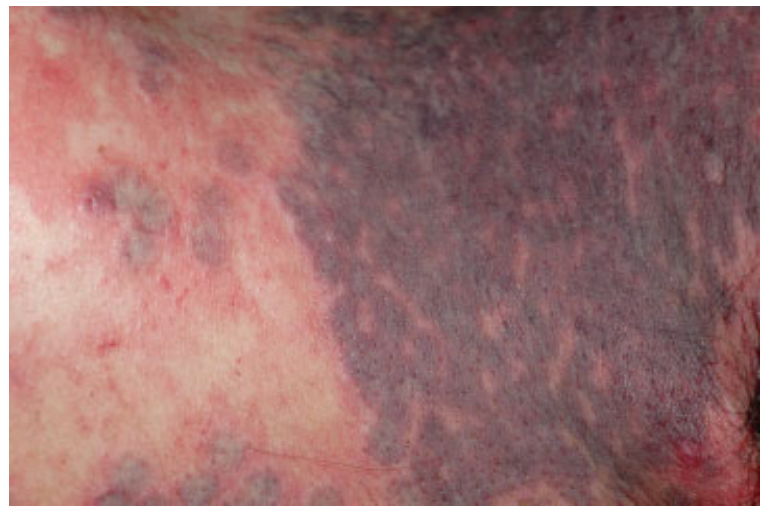

Figure 7 PWS several minutes after treatment with PDL. Note the steel-gray purpura, which indicates that that fluence was probably higher than desired.

used successfully in cases of resistant PWS. ${ }^{40-42}$ Alexandrite $(755 \mathrm{~nm})$ or Nd:YAG $(1064 \mathrm{~nm})$ lasers delivered at high fluences with good skin cooling can be particularly useful. The 532-nm KTP laser has been used in refractory PWS, but the authors noted that the shorter wavelength resulted in a higher incidence of adverse effects due to the concurrent absorption of laser energy by epidermal melanin. ${ }^{43}$ In a recent open-label clinical trial of 17 patients, a long-pulsed Nd:YAG laser was used with fluences of 1.0, 0.8, and 0.6 times the minimum purpura dose (40 to $130 \mathrm{~J} / \mathrm{cm}^{2}$ ), pulse durations ranging from 4 to 10 milliseconds, and spot size of $7 \mathrm{~mm}$. This was compared with traditional PDL treatment (595 nm, 1.5 milliseconds, $8 \mathrm{~J} / \mathrm{cm}^{2}, 7$-mm spot size). The authors found that $\mathrm{Nd}: Y A G$ laser used at the minimum purpura dose was as effective as PDL for treating PWS. Care must be undertaken, however, to closely monitor the clinical end point due to the scarring noted in some patients treated with fluences greater than the minimum purpura dose. $^{44,45}$

\section{Cherry Angiomas}

Cherry angiomas are common, benign cutaneous vascular tumors, which commonly appear on trunk or extremities. They can occasionally bleed with minor trauma. They are most common on the trunk and proximal extremities and are often found in large numbers.

Treatment is very successful with the $595-\mathrm{nm}$ PDL. Typical parameters used are 7-mm spot size and fluences of 8 to $10 \mathrm{~J} / \mathrm{cm}^{2}$. The desired clinical end point is an immediate dusky purpuric appearance. Hypertrophic cherry angiomas may require several pulses or use of diascopy to allow complete clearance.

\section{Pyogenic Granuloma}

Pyogenic granuloma is an acquired vascular lesion. If often presents as a small, solitary, rapidly enlarging, eroded papule, which bleeds easily with trauma. It may appear at any body site but commonly presents on fingers, toes, and face. Histologically, there is inflammation surrounding a hyperproliferative superficial vasculature. Because of the varying thickness of these lesions, some may not be amenable to laser treatment. Surgical excision is recommended in these cases.

PDL has been tried with success in smaller lesions. Typical parameters are fluences of 8 to $10 \mathrm{~J} / \mathrm{cm}^{2}, 7-\mathrm{mm}$ spot size, 595-nm wavelength. Multiple passes and the use of diascopy may be necessary. The clinical end point desired is a dusky purple-gray color. Because of limited penetration into the epidermis by the PDLs, nodular or thicker lesions may require deeper-wavelength lasers with increased epidermal penetration such as the millisecond near-infrared lasers (alexandrite, diode, $\mathrm{Nd}: Y A G)$. In general, patients should be informed that multiple treatments may be required. Complications include scarring and textural changes, especially with deeper-wavelength lasers and with multiple overlapping pulses.

\section{Venous Lakes}

Venous lakes are commonly acquired venule ectasias that may present later in life. They are most commonly seen on sun-exposed skin of elderly patients such as the helix of the ears, lips, cheeks, and eyelids. Development is theorized to occur from solar damage to the vascular adventitia and dermal elastic tissue. They may enlarge and bleed with trauma. Histology reveals dilated venules composed of a thin layer of endothelial cells supported by fibrous tissue in the upper dermis.

PDLs have been used successfully, but numerous treatments or pulse stacking may be needed in some cases for complete resolution. ${ }^{3}$ A single case report of successful treatment with the IPL has been reported as well. ${ }^{46}$ Use of the longer-wavelength lasers such as the millisecond near-infrared lasers (alexandrite, diode, Nd:YAG) should be effective in treatment of venous lakes for several reasons. These include sufficient penetration into the superficial dermis due to the longer wavelength, absorption by deoxyhemoglobin, adequate pulse durations to sufficiently heat the ectatic vessels, and adequate energy to cause vessel destruction. In fact, the author has successfully treated many such lesions with the long-pulsed 800-nm diode laser with pulse durations of 30 milliseconds and fluences of 36 to $40 \mathrm{~J} / \mathrm{cm}^{2}$ without adverse effects. The clinical end point is typically immediate graying and flattening of the venous lake. Similar to treatment 
of pyogenic granulomas, complications include scarring and textural changes, especially with deeper-wavelength lasers and with multiple overlapping pulses.

\section{Leg Veins/Spider Veins of the Lower Extremities}

Leg veins are a heterogeneous group of vessels, with large diameters, thick vessel walls, location deep in the dermis, and increased hydrostatic pressures in some cases. As such, successful treatment with lasers has remained a challenge. Although sclerotherapy continues to be the gold standard treatment of leg telangiectasias due to minimal adverse effects and less pain in comparison with lasers, rapid progress in laser technologies are fast making this a viable treatment alternative. This is especially true in cases where patients are averse to needles, have had a history of adverse reactions to the sclerosants, or have mainly telangiectatic matting not amenable to sclerotherapy. Although laser treatment of leg veins was thought to be less efficacious than sclerotherapy, several recent studies comparing sclerotherapy to either long-pulsed $\mathrm{Nd}: Y A G$ or alexandrite lasers found no statistical difference in efficacy of treatment. ${ }^{47,48}$

The first-generation PDLs at $585 \mathrm{~nm}$ and 0.45 milliseconds were only effective for very smalldiameter vessels $(<0.3 \mathrm{~mm})$ because of their short pulse duration and shallow depth of penetration. Secondgeneration PDLs (595 nm, 1.5 milliseconds), ${ }^{49,50}$ pulsed 532-nm KTP, ${ }^{51} 1064-\mathrm{nm}$ Nd:YAG, ${ }^{52,53}$ and IPL sources $^{54,55}$ were found to be effective in vessels up to $1 \mathrm{~mm}$ in diameter; however, they were also found to be unpredictable with high rates of posttreatment hyper- and hypopigmentation of up to $94 \%$, which were persistent for extended periods of time. Scarring was also seen. Overall, these shorter-wavelength lasers resulted in heating and thrombosis only at the superficial layers of the vessels with poor clinical results.

Studies have recently confirmed that longerwavelength lasers such as the long-pulsed $755-\mathrm{nm}$ alexandrite, ${ }^{56}$ the $800-, 810-$, and $940-$ nm diode, ${ }^{57,58}$ and the long-pulsed 1064-nm Nd:YAG ${ }^{59}$ are effective in the treatment of leg telangiectasias and postcapillary venulectasias. A study using the 1064-nm long-pulsed Nd:YAG laser has shown successful treatment of larger-diameter vessels up to $4 \mathrm{~mm}$ with one to three treatments with varying parameters ( 1.5 to $3 \mathrm{~mm}$ spot size, 30 to 60 milliseconds pulse duration, 250 to $600 \mathrm{~J} /$ $\mathrm{cm}^{2}$ ). At 6 months follow-up, $80 \%$ of patients had greater than $75 \%$ clearing. ${ }^{59}$ One prospective study compared the Nd:YAG, the 810-nm diode, and the alexandrite laser for the treatment of leg veins 0.3 to $3 \mathrm{~mm}$ in diameter. Thirty female patients with Fitzpatrick skin types I to $\mathrm{V}$ were treated. At 3 months, greater than $75 \%$ clearance was seen in $88 \%$ of patients with the Nd:YAG, in 33\% with the alexandrite laser, and in only $29 \%$ with the $810-\mathrm{nm}$ diode laser. Adverse effects of posttreatment purpura and telangiectatic matting were seen most commonly with the alexandrite laser. ${ }^{60}$

These longer-wavelength lasers allow for deeper penetration, and the longer pulse-widths allows for selective heating of larger vessels with relative sparing of smaller vessels. With the long-pulsed 1064-nm Nd:YAG laser, keep in mind that there is limited absorption of hemoglobin at this wavelength so that higher fluences are necessary for effective treatment. Treatment with these deeper-penetrating lasers can be associated with a considerable amount of treatment discomfort or pain due to volumetric heating of tissue. Therefore, epidermal cooling devices are important to minimize this adverse effect. To date, there are no long-term controlled studies on the persistence of vessel clearance after laser treatments.

Where reflux is noted, treatment of deeper varicose veins is crucial to provide durable clearing of smaller vessels. Endovenous ablation with lasers and radiofrequency devices has become a good alternative to the more invasive procedure of vein stripping and ligation. Benefits include less time to recovery and improved cosmesis in comparison with routine surgical approaches. ${ }^{61}$ It is performed by advancing a catheter through the vein either with or without tumescent anesthesia and pulling the electrode or laser fiber out slowly while heating the surrounding red blood cells and vessel wall with either direct radiofrequency (RF) heating or laser energy targeting hemoglobin or water. In the case of RF, direct thermal heating of the vessel wall with resultant vein shrinkage or occlusion by contraction of venous wall collagen is the primary mechanism of action. In the case of lasers, the mechanism is thought to be due to photomechanical effects of targeting hemoglobin, causing steam bubble formation from red blood cell rupture with resultant intramural thermal damage. The 810-, 940-, and 980-nm diode as well as the long-pulsed 1064-nm Nd:YAG has been used. Adverse effects have been reported with both RF and laser therapy. They include burns, paresthesias, thrombophlebitis, persistent pain and tenderness, localized hematomas, and postoperative purpura. ${ }^{62-66}$ In a prospective multicenter study, the vein occlusion rate with RF was found to be $81 \%$ at 1 year and $75 \%$ at 3 years. ${ }^{67}$ A study using the $940-\mathrm{nm}$ diode laser revealed complete closure in $96 \%$ at $1 \frac{1}{2}$-year follow-up in 280 patients. ${ }^{68}$ To minimize adverse effects and posttreatment pain, a 1320-nm laser system that targets water as the chromophore was recently studied in the treatment of 24 cases of incompetent great saphenous vein. 
Preliminary results demonstrated compete closure clinically and with duplex ultrasound at 6 months follow-up in all cases. ${ }^{69}$

\section{Treatment Pearls/Practical Considerations}

A good history and physical exam are crucial in helping the laser surgeon choose the correct treatment for his or her patient (Table 1). It is important to ask about the use of medications that may increase the risk of scarring (such as isotretinoin) or purpura (such as nonsteroidal anti-inflammatory drugs). Keep in mind that many over-the-counter medications such as vitamin $\mathrm{E}$ and ginkgo may also increase the risk of purpura. Inquire about the extent of sun exposure. Those with excessive sun exposure before treatment may have limited efficacy, and those with excessive exposure after treatment are at risk for hyperpigmentation. Assess the patient's expectations for treatment. In the case of PWS, is their expectation realistic? What is their pain tolerance? Are they looking for "no downtime"? In certain cases, it may be prudent to utilize more than one laser system to obtain the optimum result. Treatment should be postponed until risks are minimized (i.e., after the tan fades or after stopping medications).

Patients generally tolerate treatment well and rarely require anesthesia. Discomfort is transient and best described as a snapping sensation on the skin. For those with lower pain thresholds or with lesions in more sensitive areas, topical or localized injections of anesthesia usually suffice. If intralesional injections are required, plain lidocaine is used, as epinephrine will cause vasoconstriction of the vessel to be treated. Cooling of the skin prior to treatments with

Table 1 Important Factors in the Evaluation of Patients with Vascular Lesions

\begin{tabular}{l} 
HISTORY \\
History of medications that may increase risk of scarring \\
(isotretinoin) or purpura (nonsteroidal anti-inflammatory drugs, \\
Warfarin, ginkgo, vitamin E) \\
History of sun exposure \\
Underlying medical disorders (history of keloids) \\
Expectations of patient (improvement/clearance, purpura/no \\
purpura, number of treatments) \\
Pain tolerance (consider anesthesia if necessary) \\
PHYSICAL EXAMINATION \\
Fitzpatrick skin type \\
Anatomic location of lesion (facial, nonfacial) \\
Depth of lesion (superficial, deep) \\
Diameter of vessel (small, large) \\
Extent of lesion (focal, diffuse) \\
\hline
\end{tabular}

ice packs or forced cool air may also help to minimize pain.

If lesions are nodular (pyogenic granulomas), hypertrophic (PWS), or associated with high-flow states (spider telangiectasias), diascopy may be helpful. Diascopy allows blanching of the superficial component and deeper penetration of the laser. Alternatively, the careful delivery of additional passes, allowing the skin to cool between the pulses, is useful in improving laser energy absorption. ${ }^{70}$ Pulses are given with enough time for the skin to cool yet still maintain thermal energy within the vessels. Heating of blood vessels in this manner induces the formation of methemoglobin, which has an absorbance 4.75 times higher than that of oxygenated hemoglobin and 20 times higher than that of deoxygenated hemoglobin. ${ }^{71-73}$ Pulse stacking without cooling can also be utilized in specific cases; however, caution is recommended as vessel overheating may result in scarring, blistering, and atrophy. Treating areas away from the facial region may have a higher risk of scarring. Therefore, it is important to reduce the fluence by $\sim 10 \%$ in areas such as the neck, chest, or periorbital region.

The most commonly seen complications in the treatment of vascular lesions is pigmentary alteration. Rarely, textural changes, blistering, or scarring may be seen, especially when treating in nonfacial regions such as the neck or chest. To minimize the risks of pigmentary changes, it is important to advise the patient to avoid sun exposure to the treated area 1 month prior to and after each treatment and to use longer-wavelength lasers based on Fitzpatrick skin type. Posttreatment care is simple and generally consists of application of ice packs to reduce postoperative stinging and petrolatum or antibiotic ointment daily for several days if crusting or blistering is seen.

\section{Conclusion}

There has been tremendous progress in the development of laser systems in recent years. Lasers have now become an essential tool for physicians who treat vascular lesions. Armed with the knowledge of the basic concepts of selective photothermolysis, the laser surgeon can now choose the appropriate laser and parameters for safe and efficacious treatment. Although considerable advances have been made, several issues persist. Leg vein treatment remains unreliable with high incidences of hyperpigmentation and pain. Some PWS lesions remain resistant to treatment. Current lasers have limited use in the treatment of darker Fitzpatrick skin types. In the future, it is likely that continued improvements will produce lasers that will reliably address these issues. 


\section{REFERENCES}

1. Anderson RR, Parrish JA. Selective photothermolysis: precise microsurgery by selective absorption of pulsed radiation. Science 1983;220:524-527

2. Nelson JS, Milner TE, Anvari B, Tanenbaum BS, Svaasand LO, Kimel S. Dynamic epidermal cooling in conjunction with laser-induced photothermolysis of port wine stain blood vessels. Lasers Surg Med 1996;19:224-229

3. Gonzalez E, Gange RW, Momtaz KT. Treatment of telangiectases and other benign vascular lesions with the $577 \mathrm{~nm}$ pulsed dye laser. J Am Acad Dermatol 1992;27(2 Pt 1): 220-226

4. Ruiz-Esparza J, Goldman MP, Fitzpatrick RE, Lowe NJ, Behr KL. Flash lamp-pumped dye laser treatment of telangiectasia. J Dermatol Surg Oncol 1993;19:10001003

5. Adrian RM, Tanghetti EA. Long pulse 532-nm laser treatment of facial telangiectasia. Dermatol Surg 1998; 24:71-74

6. Goldberg DJ, Meine JG. A comparison of four frequencydoubled Nd:YAG (532 nm) laser systems for treatment of facial telangiectases. Dermatol Surg 1999;25:463-467

7. Angermeier MC. Treatment of facial vascular lesions with intense pulsed light. J Cutan Laser Ther 1999;1:95100

8. Eremia S, Li CY. Treatment of face veins with a cryogen spray variable pulse width $1064 \mathrm{~nm} \mathrm{Nd:YAG} \mathrm{laser:} \mathrm{a}$ prospective study of 17 patients. Dermatol Surg 2002;28: 244-247

9. Clark SM, Lanigan SW, Marks R. Laser treatment of erythema and telangiectasia associated with rosacea. Lasers Med Sci 2002;17:26-33

10. Lowe NJ, Behr KL, Fitzpatrick R, Goldman M, Ruiz-Esparza J. Flash lamp pumped dye laser for rosacea-associated telangiectasia and erythema. J Dermatol Surg Oncol 1991;17: 522-525

11. Tan SR, Tope WD. Pulsed dye laser treatment of rosacea improves erythema, symptomatology, and quality of life. J Am Acad Dermatol 2004;51:592-599

12. Tanghetti E, Sherr E. Treatment of telangiectasia using the multi-pass technique with the extended pulse width, pulsed dye laser (Cynosure V-Star). J Cosmet Laser Ther 2003;5: $71-75$

13. Alam M, Dover JS, Arndt KA. Treatment of facial telangiectasia with variable-pulse high-fluence pulsed-dye laser: comparison of efficacy with fluences immediately above and below the purpura threshold. Dermatol Surg 2003;29: 681-684; discussion 685

14. Jasim ZF, Woo WK, Handley JM. Long-pulsed (6-ms) pulsed dye laser treatment of rosacea-associated telangiectasia using subpurpuric clinical threshold. Dermatol Surg 2004;30: $37-40$

15. Mark KA, Sparacio RM, Voigt A, Marenus K, Sarnoff DS. Objective and quantitative improvement of rosacea-associated erythema after intense pulsed light treatment. Dermatol Surg 2003;29:600-604

16. Taub AF. Treatment of rosacea with intense pulsed light. J Drugs Dermatol 2003;2:254-259

17. Wheeland RG, Applebaum J. Flashlamp-pumped pulsed dye laser therapy for poikiloderma of Civatte. J Dermatol Surg Oncol 1990;16:12-16
18. Haywood RM, Monk BE. Treatment of poikiloderma of Civatte with the pulsed dye laser: a series of seven cases. J Cutan Laser Ther 1999;1:45-48

19. Weiss RA, Goldman MP, Weiss MA. Treatment of poikiloderma of Civatte with an intense pulsed light source. Dermatol Surg 2000;26:823-827; discussion 828

20. Weiss RA, Goldman MP, Weiss MA. Treatment of poikiloderma of Civatte with an intense pulsed light source. Dermatol Surg 2000;26:823-827; discussion 828

21. Scheepers JH, Quaba AA. Treatment of nevi aranei with the pulsed tunable dye laser at $585 \mathrm{~nm}$. J Pediatr Surg 1995;30: 101-104

22. Geronemus RG. Treatment of spider telangiectases in children using the flashlamp-pumped pulsed dye laser. Pediatr Dermatol 1991;8:61-63

23. Jacobs AH, Walton RG. The incidence of birthmarks in the neonate. Pediatrics 1976;58:218-222

24. Smoller BR, Rosen S. Port-wine stains. A disease of altered neural modulation of blood vessels? Arch Dermatol 1986; 122:177-179

25. Geronemus RG, Ashinoff R. The medical necessity of evaluation and treatment of port-wine stains. J Dermatol Surg Oncol 1991;17:76-79

26. van der Horst CM, Koster PH, de Borgie CA, Bossuyt PM, van Gemert MJ. Effect of the timing of treatment of port-wine stains with the flash-lamp-pumped pulsed-dye laser. [see comment] N Engl J Med 1998;338:10281033

27. Hansen K, Kreiter CD, Rosenbaum M, Whitaker DC, Arpey CJ. Long-term psychological impact and perceived efficacy of pulsed-dye laser therapy for patients with port-wine stains. Dermatol Surg 2003;29: 49-55

28. Troilius A, Wrangsjo B, Ljunggren B. Patients with port-wine stains and their psychosocial reactions after photothermolytic treatment. Dermatol Surg 2000;26:190-196

29. Fitzpatrick RE, Lowe NJ, Goldman MP, Borden H, Behr KL, Ruiz-Esparza J. Flashlamp-pumped pulsed dye laser treatment of port-wine stains. J Dermatol Surg Oncol 1994; 20:743-748

30. Sommer S, Seukeran DC, Sheehan-Dare RA. Efficacy of pulsed dye laser treatment of port wine stain malformations of the lower limb. Br J Dermatol 2003;149:770775

31. Algermissen B, Berlien HP, Haas N. Basal cell carcinomas in port-wine stains treated with thorium X. Acta Derm Venereol 2004;84:475

32. Jasim ZF, Woo WK, Walsh MY, Handley JM. Multifocal basal cell carcinoma developing in a facial port wine stain treated with argon and pulsed dye laser: a possible role for previous radiotherapy. Dermatol Surg 2004;30:11551157

33. Shah M, Lewis FM, Palmer IR. Three cases of multiple basal cell carcinoma arising in port-wine stains previously treated with thorium X. Br J Dermatol 1996;135:861862

34. Wharton SM, Cole RP. Basal cell carcinomata in port wine stains. Br J Plast Surg 2001;54:156-158

35. Tan OT, Murray S, Kurban AK. Action spectrum of vascular specific injury using pulsed irradiation. J Invest Dermatol 1989;92:868-871 
36. Tan ST, Bialostocki A, Armstrong JR. Pulsed dye laser therapy for rosacea. Br J Plast Surg 2004;57:303310

37. Alora MB, Anderson RR. Recent developments in cutaneous lasers. [review] Lasers Surg Med 2000;26:108118

38. Yung A, Sheehan-Dare R. A comparative study of a 595-nm with a $585-\mathrm{nm}$ pulsed dye laser in refractory port wine stains. Br J Dermatol 2005;153:601-606

39. Laube S, Taibjee S, Lanigan SW. Treatment of resistant port wine stains with the $\mathrm{V}$ Beam pulsed dye laser. Lasers Surg Med 2003;33:282-287

40. Bjerring $\mathrm{P}$, Christiansen $\mathrm{K}$, Troilius A. Intense pulsed light source for the treatment of dye laser resistant port-wine stains. J Cosmet Laser Ther 2003;5:7-13

41. Cliff S, Misch K. Treatment of mature port wine stains with the PhotoDerm VL. J Cutan Laser Ther 1999;1:101104

42. Raulin C, Goldman MP, Weiss MA, Weiss RA. Treatment of adult port-wine stains using intense pulsed light therapy (PhotoDerm VL): brief initial clinical report. Dermatol Surg 1997;23:594-597

43. Chowdhury MM, Harris S, Lanigan SW. Potassium titanyl phosphate laser treatment of resistant port-wine stains. Br J Dermatol 2001;144:814-817

44. Yang MU, Yaroslavsky AN, Farinelli WA, et al. Longpulsed neodymium:yttrium-aluminum-garnet laser treatment for port-wine stains. J Am Acad Dermatol 2005;52(3 Pt 1): 480-490

45. Woo WK, Jasim ZF, Handley JM. Evaluating the efficacy of treatment of resistant port-wine stains with variablepulse 595-nm pulsed dye and 532-nm Nd:YAG lasers. Dermatol Surg 2004;30(2 Pt 1):158-162; discussion 162

46. Jay H, Borek C. Treatment of a venous-lake angioma with intense pulsed light. Lancet 1998;351:112

47. Coles CM, Werner RS, Zelickson BD. Comparative pilot study evaluating the treatment of leg veins with a long pulsed Nd:YAG laser and sclerotherapy. Lasers Surg Med 2002;30: 154-159

48. Levy JL, Elbahr C, Jouve E. Comparison and sequential study of long pulsed Nd:YAG 1,064 nm laser and sclerotherapy in leg telangiectasias treatment. Lasers Surg Med 2004;34:273276

49. Bernstein EF, Lee J, Lowery J, et al. Treatment of spider veins with the $595 \mathrm{~nm}$ pulsed-dye laser. J Am Acad Dermatol 1998;39(5 Pt 1):746-750

50. Reichert D. Evaluation of the long-pulse dye laser for the treatment of leg telangiectasias. Dermatol Surg 1998;24:737740

51. Fournier N, Brisot D, Mordon S. Treatment of leg telangiectases with a $532 \mathrm{~nm}$ KTP laser in multipulse mode. Dermatol Surg 2002;28:564-571

52. Adrian RM. Treatment of leg telangiectasias using a longpulse frequency-doubled neodymium:YAG laser at $532 \mathrm{~nm}$. Dermatol Surg 1998;24:19-23

53. McMeekin TO. Treatment of spider veins of the leg using a long-pulsed Nd:YAG laser (Versapulse) at $532 \mathrm{~nm}$. J Cutan Laser Ther 1999;1:179-180

54. Schroeter CA, Wilder D, Reineke $T$, et al. Clinical significance of an intense pulsed light source on leg telangiectasias of up to $1 \mathrm{~mm}$ diameter. Eur J Dermatol 1997;7:38-42
55. Green D. Photothermal removal of telangiectases of the lower extremities with the PhotodermVL. J Am Acad Dermatol 1998;38:61-68

56. Kauvar AN, Lou WW. Pulsed alexandrite laser for the treatment of leg telangiectasia and reticular veins. Arch Dermatol 2000;136:1371-1375

57. Wollina U, Konrad H, Schmidt WD, Haroske G, Astafeva LG, Fassler D. Response of spider leg veins to pulsed diode laser $(810 \mathrm{~nm})$ : a clinical, histological and remission spectroscopy study. J Cosmet Laser Ther 2003;5:154162

58. Passeron T, Olivier V, Duteil L, Desruelles F, Fontas E, Ortonne JP. The new 940-nanometer diode laser: an effective treatment for leg venulectasia. J Am Acad Dermatol 2003; 48:768-774

59. Sadick NS. Laser treatment with a 1064-nm laser for lower extremity class I-III veins employing a variable spots and pulse width parameters. Dermatol Surg 2003;29:916919

60. Eremia S, Li C, Umar SH. A side-by-side comparative study of $1064 \mathrm{~nm} \mathrm{Nd:YAG,} 810 \mathrm{~nm}$ diode and $755 \mathrm{~nm}$ alexandrite lasers for treatment of $0.3-3 \mathrm{~mm}$ leg veins. [see comment] Dermatol Surg 2002;28:224-230

61. Goldman MP. Intravascular lasers in the treatment of variose veins. J Cosmet Dermatol 2004;3:162-166

62. Proebstle TM, Gul D, Kargl A, Knop J. Endovenous laser treatment of the lesser saphenous vein with a 940-nm diode laser: early results. Dermatol Surg 2003;29:357361

63. Proebstle TM, Gul D, Lehr HA, Kargl A, Knop J. Infrequent early recanalization of greater saphenous vein after endovenous laser treatment. J Vasc Surg 2003;38:511516

64. Chang CJ, Chua JJ. Endovenous laser photocoagulation (EVLP) for varicose veins. Lasers Surg Med 2002;31:257262

65. Merchant RF, Pichot O, Myers KA. Four-year follow-up on endovascular radiofrequency obliteration of great saphenous reflux. Dermatol Surg 2005;31:129-134

66. Merchant RF, DePalma RG, Kabnick LS. Endovascular obliteration of saphenous reflux: a multicenter study. J Vasc Surg 2002;35:1190-1196

67. Nicolini P. Treatment of primary varicose veins by endovenous obliteration with the VNUS closure system: results of a prospective multicentre study. Eur J Vasc Endovasc Surg 2005;29:433-439

68. Bush RG. Regarding "Endovenous treatment of the greater saphenous vein with a $940-\mathrm{nm}$ diode laser: thrombolytic occlusion after endoluminal thermal damage by laser-generated steam bubbles". J Vasc Surg 2003;37: 242

69. Goldman MP, Mauricio M, Rao J. Intravascular 1320-nm laser closure of the great saphenous vein: a 6- to 12-month follow-up study. Dermatol Surg 2004;30:1380-1385

70. Dierickx C, Farinelli WA, Anderson RR. Multiple-pulse photocoagulation of portwine stain blood vessels with a 585-nm pulsed dye laser. Lasers Surg Med 1995;S7: 261

71. Mordon S, Brisot D, Fournier N. Using a "non-uniform pulse sequence" can improve selective coagulation with a Nd:YAG laser (1.06 um) thanks to Met-hemoglobin absorption: a clinical study on blue leg veins. Lasers Surg Med 2003; $32: 160-170$ 
72. Alves OC, Wajnberg E. Heat denaturation of methemoglobin and oxyhemoglobin. Evidence for the existence of a new hemochrome. Int J Biol Macromol 1993;15:273279
73. Kuenster J, Nerris K. Spectrophotometry of human hemoglobin in the near infrared region from 1000 to $2500 \mathrm{~nm}$. J Near Infrared Spectrosc 1994;2:59-65 